\title{
Reply to the Letter to the Editor on "Helicobacter Pylori Does Not Affect Postoperative Outcomes after Sleeve Gastrectomy"
}

\author{
Hiba Shanti $^{1}$ - Neyaf Almajali ${ }^{1}$ Tamador Al-Shamaileh ${ }^{1}$ - Walid Samarah ${ }^{2}$. \\ Ayman Mismar $^{1} \cdot$ Firas Obeidat $^{1}$
}

Published online: 28 June 2017

(C) Springer Science+Business Media, LLC 2017

We thank Dr. Kassir and his colleagues for their attention to our manuscript entitled: Helicobacter pylori does not affect postoperative outcomes after sleeve gastrectomy [1].

First, we would like to focus that our study included patients undergoing sleeve gastrectomy (LSG) and not gastric bypass. Hence, the stomach is easily accessible for endoscopic surveillance postoperatively.

We fully understand your concerns and agree that Helicobacter pylori (HP) is a well-established risk factor for gastric cancer, and it is implicated in the pathogenesis of various benign and malignant diseases. Our article discussed the timing of treatment and is not against HP eradication. This issue was evident by treating all HP-positive patients in our study two weeks postoperatively.

Some papers cited in our article suggested that HP should be eradicated before surgery as it might increase the incidence of leak. However, our article showed that preoperative surveillance might not be necessary as HP infection did not affect the rate of early postoperative complications. Therefore, eradication therapy can be given postoperatively based on histopathological examination of excised stomach, which is more accurate than other methods of testing.

Worth mentioning that Keren et al. [2] showed that LSG might lead to HP eradication, due to the resection of the usual

Hiba Shanti

hibashanti@gmail.com

1 Department of General Surgery, Faculty of Medicine, University of Jordan, Queen Rania Str, P.O Box 13046, Amman 11942, Jordan

2 Department of Anesthesia, Faculty of Medicine, University of Jordan, Amman, Jordan sites of bacterial infection. This might suggest repeat testing for HP three months postoperatively before giving eradication therapy.

With regard to GERD and Barret esophagus (BE) post sleeve gastrectomy, studies had shown controversial results. Technical considerations might be implicated in the incidence of GERD postoperatively. Data from the literature concerning the development of BE after LSG are scarce. Genco et al. [3] showed a very high incidence of BE in LSG patients. Thus, endoscopic evaluation in the postoperative surveillance of LSG patients should be encouraged.

\section{Compliance with Ethical Standards}

Conflict of Interest Statement All authors declare that they have no conflicts of interest.

Statement of Human and Animal Rights All procedures performed in studies involving human participants were in accordance with the ethical standards of the institutional and/or national research committee and with the 1964 Helsinki declaration and its later amendments or comparable ethical standards.

Statement of Informed Consent Informed consent is not required for this type of study.

\section{References}

1. Shanti H, Almajali N, Al-Shamaileh T, et al. Helicobacter pylori does not affect postoperative outcomes after sleeve gastrectomy. Obes Surg. 2017;27(5):1298-301.

2. Keren D, Matter I, Rainis T, et al. Sleeve gastrectomy leads to Helicobacter pylori eradication. Obes Surg. 2009;19:751-6.

3. Genco A, Soricelli E, Casella G, et al. Gastroesophageal reflux disease and Barrett's esophagus after laparoscopic sleeve gastrectomy: a possible, underestimated long-term complication. Surg Obes Relat Dis. 2017;13(4):568-74. 